On the numerical integration of a class of pressure-dependent plasticity models including kinematic hardening

First published in:

Computational Mechanics

Vol. 31 (2003), pp. 479-488.

(C) Springer-Verlag 2003

All rights reserved.

- Reprints -

Authors:

U. Mühlich

W. Brocks 


\title{
On the numerical integration of a class of pressure-dependent plasticity models including kinematic hardening
}

Uwe Mühlich, Wolfgang Brocks (GKSS, e-mail: wolfgang.brocks@ gkss.de)

\begin{abstract}
The algorithm proposed by Aravas to integrate a special type of elastic-plastic constitutive equations has been extended to incorporate kinematic hardening. Like in the case of isotropic hardening, the number of primary unknowns for the Newton iteration can be reduced to two scalar strain variables. Furthermore, the consistent tangent can be obtained explicitly. The modified algorithm has been applied to a Gurson-type model which takes into account kinematic hardening and the predictions of the Gurson-like model are compared with results obtained by unit cell calculations.
\end{abstract}

